\title{
Do Cardiological Characteristics Explain the Mortality Rate Disparity Between Genders in COVID-19?
}

\section{Timor Omar1, ๑ Muammer Karayakalı1, ๑ Gökhan Perincek²}

${ }^{1}$ Kars Harakani State Hospital, Clinic of Cardiology, Kars, Turkey

${ }^{2}$ Kars Harakani State Hospital, Clinic of Chest Disease, Kars, Turkey

\begin{abstract}
Objectives: The coronavirus disease-2019 (COVID-19) mortality risk in males is twice as high compared to females. The literature lacks data regarding how the cardiological parameters influence the mortality rate imbalance between genders in the setting of COVID-19. This study aims to investigate how cardiological parameters vary between genders in COVID-19 disease - a potential explanation for the increased mortality rate in males in the setting of COVID-19.

Materials and Methods: We included 458 adult patients with a confirmed diagnosis of COVID-19 disease. Demographics, comorbidities, laboratory findings, and electrocardiogram parameters were compared between males and females.

Results: Of 458, a total of 63 (14.2\%) patients died, and $82(17.9 \%)$ were followed up in the intensive care unit during the hospitalization. Although the median age between males and females looked like to be similar, the mortality rate was significantly higher among males ( $44 \%$ vs $9.5 \%, p=0.006)$. High-sensitive troponin $\mathrm{T}$, presentative of myocardial injury, was considerably higher in dead patients than survivors $(p<0.05)$; however, it did not present a significant difference between genders. Electrocardiogram features did not show substantial differences, as well.

Conclusion: This study could provide important insights into the currently mostly enigmatic issue, gender-based death disparity in COVID-19, that cardiological characteristics do not impact the death imbalance.
\end{abstract}

Keywords: COVID-19, cardiology, ECG, electrocardiogram, gender, mortality

Address for Correspondence: : Timur Omar, Kars Harakani State Hospital, Clinic of Cardiology, Kars, Turkey e-mail: tbigmurad@gmail.com ORCID: orcid.org/0000-0002-2481-0505

Received: 15.08.2021 Accepted: 02.09.2021

Cite this article as: Omar T, Karakayalı M, Perincek G. Do Cardiological Characteristics Explain the Mortality Rate Disparity Between Genders in COVID-19?.. EJCM 2021;9(3):169-177.

DOI: $10.32596 /$ ejcm.galenos.2021-08-042 


\section{Introduction}

The coronavirus disease-2019 (COVID-19) pandemic is continuing to present a grave threat to humanity. A vast number of people, particularly elders and those with comorbidities, died due to the disease, and the numbers are still increasing dramatically ${ }^{(1)}$. One of the most prominent comorbidities to increase the disease's severity and mortality is cardiovascular disease (CVD) (2). The association between CVD and COVID-19 is mutual; COVID-19 may lead to various CVD pathologies, including myocarditis, arrhythmias, and heart failure (HF), while preexisting CVD magnifies the possibility of unfavorable outcomes in patients with COVID-19(2-4).

A wealth of clinical and epidemiological data demonstrated the enhanced male-gender role in mortality $^{(5,6)}$. The COVID-19 mortality risk in men was twice as high compared to that in women ${ }^{(5)}$. Various possible issues that may be responsible for the imbalance of COVID-19 mortality between genders, ranging from lifestyles to differences in chromosomal structure, were discussed ${ }^{(7)}$. However, the exact cause has remained unrevealed. Moreover, the literature lacks data regarding how the cardiological parameters influence the mortality rate imbalance between genders in the setting of COVID-19. This study aims to investigate how cardiological parameters vary between genders in COVID-19 disease - a potential explanation for the increased mortality rate in males in the setting of COVID-19.

\section{Materials and Methods}

In this single-center retrospective study, we reviewed the records of COVID-19 patients from April $28^{\text {th }}, 2020$ to October $30^{\text {th }}, 2020$. We analyzed demographics, comorbidities, laboratory findings, and electrocardiographic parameters. We included a total of 458 adult COVID-19 patients who presented to our center. The outcome of the study population was death or discharge with a cure. All patients were tested positive for severe acute respiratory syndrome coronavirus 2 (SARS-
CoV-2). We medicated patients with 5-day favipiravir oral tablets.

The Ministry of Health of Turkey and Institutional Review Board of Kafkas University approved the study protocol (reference number: 80576354-05099/277, approval date: 25.11.2020). Demographics, clinical characteristics, and laboratory findings at the admission time were collected from the medical record. High-sensitive troponin $\mathrm{T}$ (hs- $\mathrm{TnT}$ ) value at admission was also collected from the medical record. All 12lead electrocardiograms (ECG) performed on hospital admission were analyzed by a blinded cardiologist (M.K) using a standardized comprehensive electrocardiogram (ECG) reading protocol $^{(8)}$. ECG analysis included intervals, rate, rhythm, axis, QRS morphology (including bundle branch block), and ST or T-wave abnormalities. Corrected QT interval (QTc) was calculated using the Hodge formula. Demographics, comorbidities, laboratory findings, and ECGs parameters were compared between males and females.

The inclusion criteria were: 1) age $>18$ years; 2) A definite diagnosis of COVID-19. Exclusion criteria was the presence of ST-segment elevated myocardial infarction (STEMI) and the presence of grade 2 or 3 conduction block. These patient groups were referred to another center due to our center's lack of angiography laboratory.

Chronic heart failure was defined as a left ventricle ejection fraction $<50 \%$ according to previous records, and patients with an estimated glomerular filtration rate (eGFR) $<60$ (mL/minute) were determined as chronic kidney disease.

\section{Statistical Analysis}

Data obtained from this study were evaluated by using the SPSS 20 program. Continuous data were expressed as mean \pm standard deviation; categorical data were expressed as percent (\%). Data were evaluated with the Kolmogorov-Smirnov test in terms of normal distribution. The use of an independent t-test to analyze continuous data showing normal distribution and a Mann-Whitney 
U-test for the analysis of variables not showing normal distribution was planned. Crosstabs (chi-square test) were used for the comparison of categorical data. The statistical significance level was accepted as $\mathrm{p}<0.05$.

\section{Results}

Of 458 , a total of $65(14.2 \%)$ patients died, and 82 $(17.9 \%)$ were followed up in the intensive care unit during the hospitalization. Although the median age between males and females looked like to be similar, the mortality rate was significantly higher among males (44\% vs 9.5\%, p=0.006). Demographic, clinical and

Table 1. Demographic, clinical and laboratory characteristics

\begin{tabular}{|c|c|c|c|c|}
\hline & $\begin{array}{l}\text { Overall } \\
(n=458)\end{array}$ & $\begin{array}{l}\text { Male } \\
(n=238)\end{array}$ & $\begin{array}{l}\text { Female } \\
(n=220)\end{array}$ & p-value \\
\hline Gender, n (\%) & & $238(52)$ & $220(48)$ & 0.4 \\
\hline Age (years), median [IQR] & $58(36-71)$ & $55(34-71)$ & $58(39-71)$ & 0.556 \\
\hline Death, n (\%) & $65(14.2)$ & $44(18.5)$ & $21(9.5)$ & 0.006 \\
\hline Intensive care unit, $\mathbf{n}(\%)$ & $82(17.9)$ & $51(21.4)$ & $31(14.1)$ & 0.041 \\
\hline \multicolumn{5}{|l|}{ Initial vital signs } \\
\hline $\mathrm{SBP}(\mathrm{mmHg})$, median [IQR] & $120(110-120)$ & $120(110-120)$ & $120(110-130)$ & 0.054 \\
\hline DBP $(\mathrm{mmHg})$, median [IQR] & $70(60-80)$ & $70(60-80)$ & $70(60-80)$ & 0.698 \\
\hline Heart rate, median [IQR] & $84(78-96)$ & $85.5(78-99)$ & $84(78-92)$ & 0.144 \\
\hline Saturation (\%), median [IQR] & $93.5(90-95.2)$ & $93(90-96)$ & $94(90-95)$ & 0.323 \\
\hline $\mathrm{RR} /$ minute, median $[\mathrm{IQR}]$ & $20(20-22)$ & $20(20-22)$ & $20(20-22)$ & 0.088 \\
\hline \multicolumn{5}{|l|}{ Symptoms at arrival } \\
\hline Symptomatic, n (\%) & $408(89.1)$ & $210(88.2)$ & $198(90)$ & 0.545 \\
\hline Fever, n (\%) & $88(19.2)$ & $51(21.4)$ & $37(16.8)$ & 0.211 \\
\hline Cough, n (\%) & $163(35.6)$ & $94(39.5)$ & $69(31.4)$ & 0.069 \\
\hline Dyspnea, n (\%) & $148(32.3)$ & $79(33.2)$ & $69(31.4)$ & 0.676 \\
\hline Fatigue, n (\%) & $131(28.6)$ & $65(27.3)$ & $66(30)$ & 0.525 \\
\hline Nausea, n (\%) & $40(8.8)$ & $18(7.6)$ & $22(10)$ & 0.348 \\
\hline Diarrhoea, n (\%) & $10(2.2)$ & $3(1.3)$ & $7(3.2)$ & 0.16 \\
\hline Anosmia, n (\%) & $11(2.4)$ & $3(1.3)$ & $8(3.6)$ & 0.099 \\
\hline Anorexia, n (\%) & $25(5.5)$ & $8(3.4)$ & $17(7.8)$ & 0.038 \\
\hline Ageusia, n (\%) & $12(2.6)$ & $3(1.3)$ & $9(4.1)$ & 0.058 \\
\hline Sore throat, $\mathrm{n}(\%)$ & $59(12.9)$ & $28(11.8)$ & $31(14.1)$ & 0.458 \\
\hline Chest pain, n (\%) & $55(12)$ & $32(13.4)$ & $23(10.5)$ & 0.334 \\
\hline Abdominal pain, $\mathrm{n}(\%)$ & $19(4.1)$ & $7(2.9)$ & $12(5.5)$ & 0.178 \\
\hline Headache, n (\%) & $73(16)$ & $35(14.8)$ & $38(17.3)$ & 0.465 \\
\hline arthralgia/myalgia, n (\%) & $79(17.2)$ & $38(16)$ & $41(18.6)$ & 0.45 \\
\hline
\end{tabular}

laboratory characteristics are summarized in Table 1. For patients followed up in the intensive care unit, males showed a significantly higher proportion than females (21.4\% vs $14.1 \%, \mathrm{p}=0.041)$. In $408(89.1 \%)$ patients, at least one COVID-19 related symptom, was observed. The most common presented symptom was cough (35.6\%), followed by dyspnea $(32.3 \%)$ and fatigue $(28.6 \%)$. It is worthy of note that overall symptoms did not demonstrate a significant difference between genders.

Although most laboratory results showed a significant difference between genders, the medians were in normal ranges. Hs-TnT was significantly higher in dead patients 
Table 1. continued

\begin{tabular}{|c|c|c|c|c|}
\hline $\mathrm{Hgb}(\mathrm{g} / \mathrm{dL})$, median [IQR] & $14.2(12.7-15.7)$ & $15.3(13.5-16.4)$ & $13.4(12.3-14.5)$ & $<0.001$ \\
\hline WBC $\left(\times 10^{3} / \mu \mathrm{L}\right)$, median [IQR] & $7.68(5.58-7.68)$ & $8.47(6.05-12.39)$ & $6.96(5.3-10.71)$ & 0.001 \\
\hline Lymphocyte $\left(\times 10^{3} / \mu \mathrm{L}\right)$, median [IQR] & $1.5(0.92-2.06)$ & $1.45(0.83-2.01)$ & $1.57(1.06-2.14)$ & 0.042 \\
\hline PLT $\left(\times 10^{3} / \mu L\right)$, median [IQR] & $209(168-267)$ & $199(162-253)$ & $226(179-280)$ & 0.003 \\
\hline Hs-TnT(ng/L), median [IQR] & $4.97(3-12.47)$ & $6.86(3-14.54)$ & $4.06(3-9.63)$ & 0.009 \\
\hline Procalcitonin (ug/L), median [IQR] & $0.059(0.04-0.128)$ & $0.071(0.044-0.141)$ & $0.52(0.038-0.108)$ & 0.003 \\
\hline Creatinine (mg/dL), median [IQR] & $0.87(0.72-1.13)$ & $1.01(0.85-1.19)$ & $0.74(.064-0.92)$ & $<0.001$ \\
\hline D-Dimer $(\mu \mathrm{g} / \mathrm{mL})$, median [IQR] & $436(224-1086)$ & $463(217-953)$ & $421(227-1119)$ & 0.619 \\
\hline \multicolumn{5}{|l|}{ Comorbidities } \\
\hline Coronary artery disease, $\mathrm{n}(\%)$ & $42(9.2)$ & $27(11.3)$ & $15(6.8)$ & 0.094 \\
\hline chronic heart failure, n (\%) & $21(4.6)$ & $14(5.9)$ & $7(3.2)$ & 0.167 \\
\hline COPD, n (\%) & $123(26.9)$ & $70(29.4)$ & $53(24.1)$ & 0.199 \\
\hline CKD (eGFR <60 mL/min/m²), n (\%) & $22(4.8)$ & $13(5.5)$ & $9(4.1)$ & 0.493 \\
\hline Cancer, n (\%) & $10(2.2)$ & $6(2.5)$ & $4(1.8)$ & 0.607 \\
\hline Previous hypothyroidism, n (\%) & $12(2.6)$ & $3(1.3)$ & $9(4.1)$ & 0.058 \\
\hline Cerebrovascular disease, $\mathrm{n}(\%)$ & $15(3.3)$ & $6(2.5)$ & $9(4.1)$ & 0.346 \\
\hline Previous atrial fibrillation, $\mathrm{n}(\%)$ & $13(2.8)$ & $3(1.3)$ & $10(4.5)$ & 0.034 \\
\hline
\end{tabular}

than survivors $(\mathrm{p}<0.05)$; however, it did not present a significant difference between genders. Procalcitonin (normal rage $<0.5 \mathrm{ug} / \mathrm{L}$ ) was significantly higher in females than males [median (interquartile range), 0.071 $(0.044-0.141)$ vs $0.52(0.038-0.108)]$ (Table 1$)$. The most common comorbidity presented in our data was hypertension in 157 patients (34.3\%), followed by COPD in 123 patients $(26.9 \%)$. Frequencies of comorbidities were similar between genders except for previous atrial fibrillation ( $1.3 \%$ in males vs $4.5 \%$ in females, $\mathrm{p}=0.034)$. The smoking ratio was significantly higher among males than females ( $37 \%$ vs $14.1 \%, \mathrm{p}<0.001)$.

ECG of 313 participants was available. The electrocardiographical features are summarized in Table 2.
For electrocardiographic (ECG) parameters, dead patients had longer QRS intervals and more frequent ST-segment/ T-wave changes than surviving patients (both p-value, $<0.05$ ). Bundle branch block and early repolarization showed higher proportions in males, while the frequency of ST-segment/T-wave change was higher for females. QRS interval was higher, and QTc was lower among males compared to females. It is noteworthy that QTc and QRS medians were in normal ranges in both genders. All remaining ECG features were similar between genders. Farther, when a comparison was made covering only deceased patients, ECG features and hs-TnT level did not show significant difference between males and females (Table 3). 
Table 2. Electrocardiographic characteristics

\begin{tabular}{|c|c|c|c|c|}
\hline & Overall $(n=313)$ & Male $(n=164)$ & Female $(n=149)$ & p-value \\
\hline Sinus, n (\%) & $301(96.8)$ & $161(98.2)$ & $142(95.3)$ & 0.15 \\
\hline Atrial fibrillation/flutter, $\mathrm{n}(\%)$ & $10(3.2)$ & $3(1.8)$ & $7(4.7)$ & 0.34 \\
\hline Axis change, $\mathrm{n}(\%)$ & $73(23.3)$ & $45(27.4)$ & $28(18.8)$ & 0.071 \\
\hline Heart rate (b.p.m), median [IQR] & $82(72-91)$ & $80(72-90)$ & $83(72-92)$ & 0.286 \\
\hline PR interval (ms), median [IQR] & $150(130-150)$ & $150(130-166)$ & $148(130-160)$ & 0.962 \\
\hline QRS interval (ms), median [IQR] & $90(84-98)$ & $90(85-100)$ & $88(80-95)$ & $<0.001$ \\
\hline QTc (ms), mean [SD] & $429(37)$ & $422(38)$ & $436(35)$ & 0.001 \\
\hline ST-segment/T-wave change, $\mathrm{n}(\%)$ & $119(38)$ & $52(31.7)$ & $67(45)$ & 0.016 \\
\hline fragmented QRS, n (\%) & $98(31.3)$ & $54(32.9)$ & $44(29.5)$ & 0.518 \\
\hline Isolated S1Q3T3 pattern, n (\%) & $22(7.1)$ & $13(8)$ & $9(6)$ & 0.505 \\
\hline
\end{tabular}

Table 3. Comparison of electrocardiographic characteristics and hs-TnT level in deceased patients.

\begin{tabular}{|c|c|c|c|c|}
\hline & $\begin{array}{l}\text { Overall } \\
(n=43)\end{array}$ & $\begin{array}{l}\text { Male } \\
(n=28)\end{array}$ & $\begin{array}{l}\text { Female } \\
(n=15)\end{array}$ & p-value \\
\hline Atrial fibrillation/flutter, $\mathrm{n}(\%)$ & $6(9.2)$ & $2(4.5)$ & $4(19)$ & 0.08 \\
\hline Axis change $(n=43), n(\%)$ & $15(34.9)$ & $8(28.6)$ & $7(46.7)$ & 0.318 \\
\hline PR interval (ms), median [IQR] & $160(130-178)$ & $155(129-176)$ & $165(137-176)$ & 0.320 \\
\hline P-wave dispersion (ms), median [IQR] & $40(35-60)$ & $40(37-60)$ & $50(32-60)$ & 0.949 \\
\hline QRS interval (ms), median [IQR] & $95(86-100)$ & $96(87-108)$ & $95(87-98)$ & 0.277 \\
\hline QTc (ms), mean [SD] & $431(56)$ & $420(54)$ & $451(54)$ & 0.089 \\
\hline ST-segment/T-wave change, $\mathrm{n}(\%)$ & $26(60.5)$ & $15(53.6)$ & $11(73.3)$ & 0.326 \\
\hline fragmented QRS, n (\%) & $14(32.6)$ & $11(39.3)$ & $3(20)$ & 0.308 \\
\hline Isolated S1Q3T3 pattern, n (\%) & $6(14)$ & $6(21.4)$ & $0(0)$ & 0.076 \\
\hline Bandle branch block, n (\%) & $11(25.6)$ & $9(32.1)$ & $2(13.3)$ & 0.276 \\
\hline Premature atrial/ventricular contraction, $\mathrm{n}(\%)$ & $8(18.6)$ & $4(14.3)$ & $4(26.7)$ & 0.419 \\
\hline Early repolarization, $\mathrm{n}(\%)$ & $2(4.7)$ & $1(3.6)$ & $1(6.7)$ & 0.999 \\
\hline hs-TnT (ng/L), median [IQR] $(n=14)$ & $45(31-83)$ & $41(38-70)$ & $50(20-83)$ & 0.999 \\
\hline
\end{tabular}




\section{Discussion}

Here, to our knowledge, we presented the first study addressing the role of cardiological characteristics in sex-dependent death-disparity in COVID-19. Our study revealed that the mortality rate was twice higher among males than females in a similar line with previous reports $^{(5,9)}$. The majority of the patients who were followed up in the intensive care unit (ICU), which indicated disease severity, were males. When comparing males and females, initial vital signs, symptoms, laboratory results, and comorbidities showed similar features. Additionally, when the cardiological characteristics, including hs$\mathrm{TnT}$, the indicator of cardiac injury, were compared, we could not find a significant difference between genders. However, the smoking rate was higher among males.

A vast number of reports showed the striking dominance of male sex, around twice as high, in the overall mortality in patients with COVID-19(5,10). The most extensive study to date, OpenSAFELY ${ }^{(5)}$, has assessed data from over 17 million patients in the United Kingdom and identified that males have over twice the as high increased risk of death as females. Another data from five European countries (France, Italy, Spain, Switzerland, and Germany) presented a similar outcome ${ }^{(6)}$. These critical reports confirmed an imbalance of COVID-19 severity, hospitalization, and mortality between men and women.

A range of hypotheses, from lifestyles to differences in chromosomal structure, from underlying comorbidities to variation in sex hormones, have been argued regarding gender-based death disparity in the context of COVID-19.

According to prior reports, for cardiological characteristics, there were various ECG abnormalities associated with poor outcomes in patients with COVID-19 ${ }^{(11,12)}$. In a study, ventricular arrhythmia and sinus tachycardia were associated with higher mortality in COVID-19 patients ${ }^{(13)}$. Another study highlighted that prolonged QTc was an independent risk factor of mortality in COVID-19 ${ }^{(14)}$. Romero et al. ${ }^{(15)}$ found out that $\mathrm{T}$ wave inversion was also associated with mortality in the setting of COVID-19 disease. Furthermore, according to Abrams et al. ${ }^{(16)}$, longer QTc and left bundle branch block on admission showed an increased risk of death. For ECG changes in the current study, dead patients had longer QRS intervals, more frequent ST-segment/Twave changes than surviving patients. However, when a comparison was made, these ECG features did not show a significant difference between males and females. Bundle branch block and early repolarization showed higher proportions in males. However, it is hard to link just the two ECG changes to the increased mortality; while, critical ECG abnormalities such as fragmented QRS, QTC, ST-segment/T-wave changes showed no difference between genders.

According to previous reports, myocardial injury, which was defined as troponin value above the $99^{\text {th }}$ percentile upper reference limit, was directly correlated with increased mortality in patients with COVID-9 ${ }^{(17)}$. Similarly, in our study, the median values of hs-TnT at admission were markedly higher in the dead patients than in the survivors. Nevertheless, it showed similar medians between genders.

COVID-19 patients with underlying comorbidities in both young and older individuals show more severe symptoms and higher mortality irrespective of gender ${ }^{(18,19)}$. A field that has pioneered this dialogue is underlying cardiovascular disease (CVD) ${ }^{(2,20)}$. Past evidence presented that patients with a history of CVD met with unfavorable outcomes of COVID-19 more frequently ${ }^{(21)}$. The current study showed similar proportions of CVD in both genders. It can be suggested that CVD did not influence male-based increased mortality in patients with COVID- 19.

We also assessed the impact of CVD risk factors such as hypertension, advanced age, and smoking. Arterial hypertension, which is clearly a male-biased disease, is an excellent example of a sex-based death imbalance in COVID-19(22) . A retrospective observational study by Gao et al. ${ }^{(23)}$ has observed a two-fold increase of COVID-19 mortality in hypertensive individuals, highlighting a clear relationship between hypertension and COVID-19. However, the proportion of hypertension was similar in both genders in our data. 
Older age, another classical CVD risk factor, is most profoundly implicated in COVID-19-associated mortality. The OpenSAFELY study has identified that hazard ratios increase with age, increasing from 2.40 in those over 60 years old to 20.61 in individuals over 80 years old ${ }^{(5)}$. Similarly, in our study, the mortality rate was positively correlated with advanced age. However, notably, the median age in both genders showed no difference.

Ahmed et al.$^{(9)}$ argued the role of smoking in COVID-19 in a commentary. They suggested that men could potentially have poorer respiratory outcomes in SARSCov-2 infection because they were more likely to smoke. They also highlighted that this remained speculative as gender-stratified smoking rates were not specifically reported in the published COVID-19 literature. When we looked at our data, the percentage of smoking was higher among males in comparison to females. Also, smoking was significantly higher in dead patients than in those who survived. Additionally, when a comparison was made among only dead patients, the smoking ratio was higher in males than in females. Herein, could smoking, as a cardiovascular disease risk factor, explain the male-based mortality rise in COVID-19? Possible, but more evidence is needed in this field.

As for the other arguments that potentially help explain the high mortality of males in the context of COVID-19, many genes that play crucial roles in immune responses are present on the $\mathrm{X}$ chromosome. Current evidence demonstrates that the differential expression and regulation of X-linked genes between males and females play significant roles in sexual dimorphic responses to infection ${ }^{(24)}$. Furthermore, the sex-related difference in the immune system, sex hormone milieu, and other unknown causes may contribute to the high mortality of males in stressful conditions, including COVID-19 ${ }^{(25)}$. According to some reports, sex and sex hormones affect many components of the circulating and tissue-based renin-angiotensin-aldosterone system (RAAS), including ACE2 ${ }^{(26)}$. Nevertheless, reports from several preclinical studies agree that ACE2 is frequently higher in males than in females, mainly under pathological conditions ${ }^{(27,28)}$. It is a point of question whether this influences death disparity between genders.

Ultimately, numerous reports, with various hypotheses, tried to explain the higher mortality rate in males than in females in the setting of COVID-19. However, the exact cause has remained unclear.

In this study, we focused on the potential cardiological influence on the sex-based disparity for COVID-19 deaths. However, we did not meet any cardiological characteristics including ECG parameters and hs-TnT and comorbidities such as hypertension, diabetes, chronic heart failure, chronic kidney disease (CKD), cancer, hypothyroidism, COPS, CVD, cerebrovascular disease, and age, which contribute to the increased mortality rate among males. Future studies should explore the effects of additional factors on COVID-19 susceptibility/mortality disparity within the context of sex groups to disentangle these issues.

\section{Study Limitations}

This study has several significant limitations that must be acknowledged: 1) we did not have previous ECGs of patients and, thus, we could not distinguish between newonset vs. chronic ECG abnormalities, 2) we only assessed ECGs which were available on admission; herewith, we cannot exclude that a later cardiac involvement with subsequent ECG changes might have resulted in some differences between the groups, 3) This study lacks echocardiographic imaging; echocardiographic workup in patients with COVID-19 was often challenging due to efforts to mitigate exposure risk of health care workers, 4) Since we were lack of detailed data about pharmacological therapy for comorbidities, we could not calculate how treatment affected survival, in-depth. Longterm observations and prospective studies are needed.

\section{Conclusion}

This study could provide important insights into the currently mostly enigmatic issue, gender-based death 
disparity in COVID-19, that cardiological characteristics do not impact the death imbalance. More comprehensive studies are needed in this area.

\section{Ethics}

Ethics Committee Approval: Institutional Review Board of Kafkas University approved the study protocol (reference number: 80576354-050-99/277, approval date: 25.11.2020).

Informed Consent: Not required (retrospective study).

Peer-review: Externally peer-reviewed.

\section{Authorship Contributions}

Conception and/or Design: T.O., Acquisition of Data: M.K., G.P., Analysis and/or Interpretation of Data: T.O., Drafting of the Manuscript: T.O., Critical Revision: M.K., Statistical Analysis: T.O., Supervision: M.K.

Conflict of Interest: The authors declared no potential conflicts of interest with respect to the research, authorship, and/or publication of this article.

Financial Disclosure: The authors received no financial support for the research, authorship, and/or publication of this article.

\section{References}

1. World Health Organisation (WHO) Available at: https://www.who.int/ emergencies/diseases/novel-coronavirus-2019 (Accessed: 15.07.2021).

2. Guzik TJ, Mohiddin SA, Dimarco A, et al. COVID-19 and the cardiovascular system: implications for risk assessment, diagnosis, and treatment options. Cardiovasc Res 2020;116:1666-87.

3. Fried JA, Ramasubbu K, Bhatt R, et al. The Variety of Cardiovascular Presentations of COVID-19. Circulation 2020;141:1930-6.

4. South AM, Diz DI, Chappell MC. COVID-19, ACE2, and the cardiovascular consequences. Am J Physiol Heart Circ Physiol 2020;318:H1084-90.

5. Williamson EJ, Walker AJ, Bhaskaran K, et al. Factors associated with COVID-19-related death using OpenSAFELY. Nature 2020;584:430-6.

6. Gebhard C, Regitz-Zagrosek V, Neuhauser HK, Morgan R, Klein SL. Impact of sex and gender on COVID-19 outcomes in Europe. Biol Sex Differ 2020;11:29.
7. Bienvenu LA, Noonan J, Wang X, Peter K. Higher mortality of COVID-19 in males: sex differences in immune response and cardiovascular comorbidities. Cardiovasc Res 2020;116:2197-206.

8. Surawicz B, Knilans T. Chou's Electrocardiography in Clinical Practice. 6th ed. Saunders; 2008.

9. Ahmed SB, Dumanski SM. Sex, gender and COVID-19: a call to action. Can J Public Health 2020;111:980-3.

10. Bhopal SS, Bhopal R. Sex differential in COVID-19 mortality varies markedly by age. Lancet 2020;396:532-3.

11. Goyal P, Choi JJ, Pinheiro LC, et al. Clinical Characteristics of Covid-19 in New York City. N Engl J Med 2020;382:2372-4.

12. De Vita A, Ravenna SE, Covino M, et al. Electrocardiographic Findings and Clinical Outcome in Patients with COVID-19 or Other Acute Infectious Respiratory Diseases. J Clin Med 2020;9:3647.

13. Li L, Zhang S, He B, Chen X, Wang S, Zhao Q. Risk factors and electrocardiogram characteristics for mortality in critical inpatients with COVID-19. Clin Cardiol 2020;43:1624-30.

14. Farré N, Mojón D, Llagostera M, et al. Prolonged QT Interval in SARS CoV-2 Infection: Prevalence and Prognosis. J Clin Med 2020;9:2712.

15. Romero J, Alviz I, Parides M, et al. T-wave inversion as a manifestation of COVID-19 infection: a case series. J Interv Card Electrophysiol 2020;59:485-93.

16. Abrams MP, Wan EY, Waase MP, et al. Clinical and cardiac characteristics of COVID-19 mortalities in a diverse New York City Cohort. J Cardiovasc Electrophysiol 2020;31:3086-96.

17. Ghio S, Baldi E, Vicentini A, et al. Cardiac involvement at presentation in patients hospitalized with COVID-19 and their outcome in a tertiary referral hospital in Northern Italy. Intern Emerg Med 2020;15:1457-65.

18. Ge H, Wang X, Yuan X, et al. The epidemiology and clinical information about COVID-19. Eur J Clin Microbiol Infect Dis 2020;39:1011-9.

19. Han Q, Lin Q, Jin S, You L. Coronavirus 2019-nCoV: A brief perspective from the front line. J Infect 2020;80:373-7.

20. Regitz-Zagrosek V, Kararigas G. Mechanistic Pathways of Sex Differences in Cardiovascular Disease. Physiol Rev 2017;97:1-37.

21. Keri VC, Hooda A, Kodan P, R L B, Jorwal P, Wig N. Intricate interplay between Covid-19 and cardiovascular diseases. Rev Med Virol 2021;31:e2188.

22. Drummond GR, Vinh A, Guzik TJ, Sobey CG. Immune mechanisms of hypertension. Nat Rev Immunol 2019;19:517-32.

23. Gao C, Cai Y, Zhang K, et al. Association of hypertension and antihypertensive treatment with COVID-19 mortality: a retrospective observational study. Eur Heart J 2020;41:2058-66.

24. Klein SL, Flanagan KL. Sex differences in immune responses. Nat Rev Immunol 2016;16:626-38

25. Pradhan A, Olsson PE. Sex differences in severity and mortality from COVID-19: are males more vulnerable? Biol Sex Differ 2020;11:53. 
26. Patel SK, Velkoska E, Burrell LM. Emerging markers in cardiovascular disease: where does angiotensin-converting enzyme 2 fit in? Clin Exp Pharmacol Physiol 2013;40:551-9.

27. Fischer M, Baessler A, Schunkert H. Renin angiotensin system and gender differences in the cardiovascular system. Cardiovasc Res 2002;53:672-7.
28. Chappell MC, Marshall AC, Alzayadneh EM, Shaltout HA, Diz DI. Update on the Angiotensin converting enzyme 2-Angiotensin (1-7)-MAS receptor axis: fetal programing, sex differences, and intracellular pathways. Front Endocrinol (Lausanne) 2014;4:201 